\title{
Impact of chronic obstructive pulmonary disease and frailty on long- term outcomes and quality of life after transcatheter aortic valve implantation
}

\author{
Artur Dziewierz ${ }^{1}$ D $\cdot$ Tomasz Tokarek $^{2} \cdot$ Pawel Kleczynski $^{1} \cdot$ Danuta Sorysz $^{1} \cdot$ Maciej Bagienski $^{1} \cdot$ Lukasz Rzeszutko $^{1}$ \\ Dariusz Dudek ${ }^{1,2}$
}

Received: 13 October 2017 / Accepted: 17 November 2017 / Published online: 28 November 2017

(c) The Author(s) 2017. This article is an open access publication

\begin{abstract}
Background Association between chronic obstructive pulmonary disease (COPD) and long-term mortality as well as the quality of life (QoL) in patients with severe aortic stenosis (AS) undergoing transcatheter aortic valve implantation (TAVI) is still unclear.

Aim We sought to evaluate the impact of COPD on mortality and QoL of patients with AS undergoing TAVI.

Methods A total of 148 consecutive patients who underwent TAVI were enrolled and stratified by history of COPD.

Results Of 148 patients enrolled, 19 (12.8\%) patients had a history of COPD. Patients with COPD were high-risk patients with higher prevalence of incomplete revascularization and frailty features. At follow-up of 15.8 months, all-cause mortality in patients with COPD was over four times higher than in patients without COPD [17.8\% vs. $52.6 \% ; p=0.002$-age/genderadjusted OR (95\% CI) 4.73 (1.69-13.24)]. On the other hand, in Cox regression model, the only independent predictors of all-cause death at long-term follow-up were: incomplete coronary revascularization [HR (95\% CI) 5.45 (2.38-12.52); $p=0.001$ ], estimated glomerular filtration rate [per $1 \mathrm{ml} / \mathrm{min} / 1.73 \mathrm{~m}^{2}$ increase: $0.96(0.94-0.98) ; p=0.001$ ], and previous stroke/transient ischemic attack [2.86 (1.17-7.00); $p=0.021]$. Also, the difference in mortality between patients with and without COPD was not significant after adjustment for the most of frailty indices. Importantly, groups were comparable in terms of QoL at baseline and 12 months.

Conclusion COPD may pose an important factor affecting long-term outcomes of patients with severe AS undergoing TAVI. However, its effects might be partially related to coexisting comorbidities and frailty.
\end{abstract}

Keywords Transcatheter aortic valve replacement $\cdot$ Frailty $\cdot$ Aortic valve disease $\cdot$ Elderly

\section{Introduction}

Concomitant chronic obstructive pulmonary disease (COPD) in patients with severe aortic stenosis (AS) is associated with higher morbidity and mortality after surgical aortic valve replacement (SAVR) [1, 2]. Furthermore, COPD is considered as a marker of poor prognosis and is incorporated in

Artur Dziewierz

adziewierz@gmail.com

1 Second Department of Cardiology, Institute of Cardiology, Jagiellonian University Medical College, 17 Kopernika St., 31-501 Krakow, Poland

2 Department of Interventional Cardiology, Institute of Cardiology, Jagiellonian University Medical College, 17 Kopernika St., 31-501 Krakow, Poland the contemporary cardiac surgery risk prediction models $[3,4]$. This results in many elderly patients with severe AS and concomitant COPD being denied surgery and scheduled for transcatheter aortic valve implantation (TAVI) as a lower risk alternative. However, in spite of an improvement in outcomes and quality of life (QoL) after TAVI [5-12], recently, some studies reported a detrimental effect of COPD on outcomes of patients with severe AS undergoing TAVI [13-15]. Nevertheless, the impact of COPD on TAVI results seems to be still unclear $[16,17]$. Also, data on the possible associations between COPD, frailty, QoL, and outcomes for patients undergoing TAVI are scarce. Thus, we sought to evaluate the impact of COPD on long-term outcomes and QoL in patients with severe AS undergoing TAVI. 


\section{Methods}

A total of 148 consecutive patients with symptomatic severe AS who underwent TAVI were included. All the patients were considered inoperable or high-risk for conventional SAVR [8]. Baseline characteristics and procedural data were collected prospectively. Frailty indices were assessed before TAVI with the Katz index of Independence of Activities in Daily Living, elderly mobility scale (EMS) score, Canadian Study of Health and Aging (CSHA) scale, 5-meter walking test, dominant hand grip strength, and Identification of Seniors at Risk scale [18]. Hand grip strength assessment was performed by 1 physician and it was subjectively distinguished into the following: weak, mild and strong. It was performed using patient's dominant hand and patients were asked to squeeze dominant hand as tightly as possible (repeated twice) [18]. TAVI procedures were performed using Edwards Sapien, Edwards Sapien XT, Edwards Sapien 3 (Edwards Lifesciences), Medtronic Core Valve/Evolut R (Medtronic, Inc), JenaValve (JenaValve Technology), Lotus (Boston Scientific) and NVT (New Valve Technology). The decision on type and size of the valve as well as access route was at the discretion of the Heart Team and treating physicians. Procedures were performed under general or local anesthesia with mild sedation. Clinical endpoints of the study included all-cause mortality at 30 days and every 6 months up to maximal available follow-up and complications rate up to 12 months. Outcome end points were in accordance with Valve Academic Research Consortium definitions (VARC-2) [19]. QoL was assessed with the self-reported Polish validated version of the EQ-5D-3L questionnaire at baseline and at 12 months after TAVI. The EQ-5D-3L is a health-related QoL measure, consisting of 5 three-level items, representing various aspects of health: mobility, self-care, usual activities, pain/discomfort and anxiety/depression. Respondents can score each domain from one (no problems) to three (extreme problems). The visual analog scale (VAS) score, which is a part of the EQ-5D-3L, was also assessed. For the analysis, patients were stratified by history of COPD as patients with and without concomitant COPD. The diagnosis of COPD was based on the medical history and the results of pulmonary function tests [20]. Spirometry was an obligatory part of the diagnostic work-up for all patients scheduled for TAVI. Standard reference values were used.

Results are presented as number of patients (percentage) or median (interquartile range [IQR]) where applicable. Continuous variables were compared with Mann-Whitney $U$ test. Categorical variables were compared with the Chi square test or Fisher's exact test. Changes in the proportions of patients who reported either "no problems" or "some problems"/"extreme problems" on the EQ-5D-3L between baseline and follow-up visits were analyzed using McNemar's test. Differences in the VAS score between baseline and follow-up assessments were analyzed with a Wilcoxon signed-rank test. All comparisons between baseline and 12-month measurements were performed excluding unpaired results. Survival curves were constructed using Kaplan-Meier estimates and compared using the log-rank test. Differences in outcomes are presented as adjusted for age/gender odds ratios (OR) with confidence intervals (95\% CI). In addition, a multivariable Cox proportional hazards model including baseline variables (except for frailty indices) was used to identify predictors of all-cause mortality at maximal follow-up. Forward selection with a probability value for covariates to enter the model was set at the 0.05 level. Then, additional Cox regression models adjusted for age/gender were constructed to assess associations between COPD, frailty and maximal follow-up all-cause mortality. Results are presented as hazard ratios (HR) with associated $95 \%$ CI. A 2 -sided $p$ value $<0.05$ was considered statistically significant. All analyses were performed using SPSS 15.0 (SPSS, Inc, Chicago, IL, USA).

\section{Results}

A total of 148 consecutive patients who underwent TAVI were enrolled. Of them, 19 (12.8\%) patients had a history of COPD. Patients with COPD were high-risk individuals with higher prevalence of diabetes mellitus, previous myocardial infarction (MI) and percutaneous coronary intervention as compared to patients without COPD (Table 1). In addition, patients with COPD were more likely to have incomplete coronary revascularization before TAVI. The prevalence of frailty features as assessed with EMS, CSHA, and Katz indices was numerically higher in patients with than without COPD. The grip strength in patients with COPD was lower than in patients without COPD. However, no difference in the prevalence of 'weak' category between groups was observed (Table 2). Despite some dissimilarities in baseline risk profile, there were no differences regarding procedural technique and echocardiographic outcomes of TAVI between patients with and without COPD (Table 3).

The median length of hospital stay was comparable between groups [COPD (-) vs. COPD (+): $10.0(8.0-13.0)$ vs. $9.5(7.0-12.0)$ days; $p=0.50]$. No difference in the rate of bleeding [32.6\% vs. $36.8 \% ; p=0.71$, age/gender-adjusted OR (95\% CI) $1.23(0.44-3.39)]$ as well as blood transfusion [29.5\% vs. $31.6 \% ; p=0.85$-age/gender-adjusted OR $(95 \%$ CI) 1.12 (0.39-3.23)] during hospital stay was observed. Similarly, the rate of grade 3 acute kidney injury (AKI) was comparable between groups [5.4\% vs. $5.4 \% ; p=1.00$-age/ 
Table 1 Baseline characteristics and echocardiographic data

\begin{tabular}{|c|c|c|c|c|}
\hline \multirow[t]{2}{*}{ Variable } & \multirow[t]{2}{*}{ All patients $(n=148)$} & \multicolumn{2}{|c|}{$\begin{array}{l}\text { Chronic obstructive pulmonary } \\
\text { disease }\end{array}$} & \multirow[t]{2}{*}{$p$ value } \\
\hline & & Absent $(n=129)$ & Present $(n=19)$ & \\
\hline Age, median (IQR) [years] & $82.0(77.0-85.0)$ & $82.0(78.0-85.0)$ & $78.0(75.5-83.5)$ & 0.26 \\
\hline Age $\geq 80$ years & $92(62.2 \%)$ & $83(64.3 \%)$ & $9(47.4 \%)$ & 0.15 \\
\hline Men & $56(37.8 \%)$ & $46(35.7 \%)$ & $10(52.6 \%)$ & 0.15 \\
\hline Body mass index, median (IQR) $\left[\mathrm{kg} / \mathrm{m}^{2}\right]$ & $27.2(25.2-30.6)$ & $27.3(25.3-30.2)$ & $28.1(25.8-33.8)$ & 0.39 \\
\hline Estimated glomerular filtration rate, median (IQR) $\left[\mathrm{ml} / \mathrm{min} / 1.73 \mathrm{~m}^{2}\right]$ & $56.5(40.0-72.0)$ & $54.0(39.0-72.0)$ & $66.0(45.0-80.0)$ & 0.19 \\
\hline New York Heart Association class & & & & 0.24 \\
\hline I & $0(0.0 \%)$ & $0(0.0 \%)$ & $0(0.0 \%)$ & \\
\hline II & $41(27.7 \%)$ & $36(27.9 \%)$ & $5(26.3 \%)$ & \\
\hline III & $97(65.5 \%)$ & $86(66.7 \%)$ & $11(57.9 \%)$ & \\
\hline IV & $10(6.8 \%)$ & $7(5.4 \%)$ & $3(15.8 \%)$ & \\
\hline Arterial hypertension & $139(93.9 \%)$ & $120(93.0 \%)$ & $19(100.0 \%)$ & 0.61 \\
\hline Diabetes mellitus & $48(32.4 \%)$ & $38(29.5 \%)$ & $10(52.6 \%)$ & 0.044 \\
\hline Atrial fibrillation & $52(35.1 \%)$ & $44(34.1 \%)$ & $8(42.1 \%)$ & 0.50 \\
\hline Previous myocardial infarction & $48(32.4 \%)$ & $38(29.5 \%)$ & $10(52.6 \%)$ & 0.044 \\
\hline Previous percutaneous coronary intervention & $43(29.1 \%)$ & $33(25.6 \%)$ & $10(52.6 \%)$ & 0.015 \\
\hline Previous coronary artery bypass grafting & $28(18.9 \%)$ & $26(20.2 \%)$ & $2(10.5 \%)$ & 0.53 \\
\hline Chronic total occlusion & $14(9.5 \%)$ & $8(6.2 \%)$ & $6(31.6 \%)$ & 0.003 \\
\hline Incomplete revascularization & $22(14.9 \%)$ & $15(11.6 \%)$ & $7(36.8 \%)$ & 0.010 \\
\hline Previous stroke/transient ischemic attack & $17(11.5 \%)$ & $13(10.1 \%)$ & $4(21.1 \%)$ & 0.24 \\
\hline Pacemaker & $17(11.5 \%)$ & $17(13.2 \%)$ & $0(0.0 \%)$ & 0.13 \\
\hline Logistic Euroscore I, median (IQR) [\%] & $14.5(10.0-22.7)$ & $14.0(10.0-22.0)$ & $15.0(13.4-23.5)$ & 0.10 \\
\hline The Society of Thoracic Surgeons score, median (IQR) [\%] & $6.2(4.0-17.3)$ & $7.0(4.0-17.0)$ & $5.5(3.8-30.0)$ & 0.65 \\
\hline Maximal transvalvular gradient, median (IQR) $[\mathrm{mmHg}]$ & $86.0(69.0-103.0)$ & $87.0(70.0-104.0)$ & $80.0(71.0-90.5)$ & 0.21 \\
\hline Mean transvalvular gradient, median (IQR) $[\mathrm{mmHg}]$ & $50.0(42.0-63.0)$ & $50.0(42.0-64.5)$ & $47.0(42.5-53.0)$ & 0.37 \\
\hline Aortic valve area, median (IQR) $\left[\mathrm{cm}^{2}\right]$ & $0.7(0.6-0.8)$ & $0.7(0.5-0.8)$ & $0.7(0.6-1.0)$ & 0.09 \\
\hline Left ventricle ejection fraction, median (IQR) [\%] & $60.0(50.0-65.0)$ & $60.0(50.0-65.0)$ & $60.0(49.5-65.0)$ & 0.57 \\
\hline Aortic regurgitation & & & & 0.034 \\
\hline 0 & $48(32.4 \%)$ & $46(35.7 \%)$ & $2(10.5 \%)$ & \\
\hline 1 & $75(50.7 \%)$ & $62(48.1 \%)$ & $13(68.4 \%)$ & \\
\hline 2 & $20(13.5 \%)$ & $18(14.0 \%)$ & $2(10.5 \%)$ & \\
\hline 3 & $5(3.4 \%)$ & $3(2.3 \%)$ & $2(10.5 \%)$ & \\
\hline Systolic pulmonary artery pressure $\geq 46 \mathrm{mmHg}$ & $65(43.9 \%)$ & $54(41.9 \%)$ & $11(57.9 \%)$ & 0.19 \\
\hline
\end{tabular}

gender-adjusted OR (95\% CI) 0.87 (0.10-7.96)]. At 30 days, no difference in all-cause mortality was observed $[7.8 \%$ vs. $10.5 \% ; p=0.65$-age/gender-adjusted OR (95\% CI) 1.37 (0.27-6.90)]. However, at 12 months all-cause mortality in patients with COPD was over 4 times higher than in patients without COPD [11.6\% vs. $36.8 \% ; p=0.010$-age/genderadjusted OR (95\% CI) 4.19 (1.34-13.09)]. This difference in mortality was maintained during the median follow-up of 15.8 (6.4-33.1) months [17.8\% vs. 52.6\%; $p=0.002$-age/ gender-adjusted OR (95\% CI) 4.73 (1.69-13.24)]-Fig. 1. On the other hand, the difference in mortality between patients with and without COPD was not significant after adjustment for most of the frailty indices (Table 4). In addition, in Cox regression model, COPD was not identified as an independent predictor of long-term all-cause mortality. The only independent predictors were: incomplete coronary revascularization [HR (95\% CI) 5.45 (2.38-12.52); $p=0.001]$, estimated glomerular filtration rate [HR $(95 \% \mathrm{CI})$ per $1 \mathrm{ml} / \mathrm{min} / 1.73 \mathrm{~m}^{2}$ increase: $\left.0.96(0.94-0.98) ; p=0.001\right]$, and previous stroke/transient ischemic attack [HR (95\% CI) $2.86(1.17-7.00) ; p=0.021]$. In addition, a trend towards higher risk of MI [1.6\% vs. $10.5 \% ; p=0.08$-age/genderadjusted OR (95\% CI) $8.52(0.94-77.55)]$, cerebrovascular accidents $[5.4 \%$ vs. $15.8 \% ; p=0.12$-age/gender-adjusted OR (95\% CI) 2.77 (0.63-12.28)], and new onset of atrial fibrillation $[5.4 \%$ vs. $15.8 \% ; p=0.012$-age/genderadjusted OR (95\% CI) $2.45(0.53-11.32)]$ at 12 months was noted in patients with COPD. New permanent pacemaker 
Table 2 Frailty indices in patients with and without chronic obstructive pulmonary disease

\begin{tabular}{|c|c|c|c|c|c|}
\hline \multirow[t]{2}{*}{ Variable } & \multirow[t]{2}{*}{ Categories } & \multirow[t]{2}{*}{$\begin{array}{l}\text { All patients } \\
(n=148)\end{array}$} & \multicolumn{2}{|c|}{$\begin{array}{l}\text { Chronic obstructive pulmonary } \\
\text { disease }\end{array}$} & \multirow[t]{2}{*}{$p$ value } \\
\hline & & & $\begin{array}{l}\text { Absent } \\
(n=129)\end{array}$ & $\begin{array}{l}\text { Present } \\
(n=19)\end{array}$ & \\
\hline 5-meter walking test (seconds) & $\geq 6$, frail & $21(14.2 \%)$ & $17(13.2 \%)$ & $4(21.1 \%)$ & 0.48 \\
\hline \multirow[t]{3}{*}{ Elderly mobility scale (points) } & $<10$, frail & $8(5.4 \%)$ & $5(3.9 \%)$ & $3(15.8 \%)$ & 0.11 \\
\hline & $10-13$ & $93(62.8 \%)$ & $83(64.3 \%)$ & $10(52.6 \%)$ & \\
\hline & $>13$ & $47(31.8 \%)$ & $41(31.8 \%)$ & $6(31.6 \%)$ & \\
\hline \multirow[t]{4}{*}{ Canadian Study of Health and Aging scale (points) } & $1-3$ & $87(58.8 \%)$ & $78(60.5 \%)$ & $9(47.4 \%)$ & 0.06 \\
\hline & 4 & $44(29.7 \%)$ & $37(28.7 \%)$ & $7(36.8 \%)$ & \\
\hline & 5 , frail & $3(2.0 \%)$ & $1(0.8 \%)$ & $2(10.5 \%)$ & \\
\hline & $6-7$, frail & $14(9.5 \%)$ & $13(10.1 \%)$ & $1(5.3 \%)$ & \\
\hline Katz index (points) & $<6$, frail & $19(12.8 \%)$ & $14(10.9 \%)$ & $5(26.3 \%)$ & 0.07 \\
\hline \multirow[t]{3}{*}{ Grip strength (grade) } & Weak, frail & $7(4.7 \%)$ & $6(4.7 \%)$ & $1(5.3 \%)$ & 0.025 \\
\hline & Mild & $14(9.5 \%)$ & $9(7.0 \%)$ & $5(26.3 \%)$ & \\
\hline & Strong & $127(85.8 \%)$ & $114(88.4 \%)$ & $13(68.4 \%)$ & \\
\hline Identification of Seniors at Risk scale (points) & $\begin{array}{r}\geq 2 \text {, functional } \\
\text { decline, frail }\end{array}$ & $53(35.8 \%)$ & $46(35.7 \%)$ & $7(36.8 \%)$ & 0.92 \\
\hline
\end{tabular}

implantation was required in $17.1 \%$ of patients without COPD and $10.5 \%$ of patients with COPD [ $p=0.74-$ age/ gender-adjusted OR (95\% CI) 0.60 (0.13-2.80)].

In general, baseline QoL parameters as assessed with the EQ-5D-3L were comparable between groups. However, a higher rate of reporting either "some problems"/“extreme problems" in terms of self-care in patients with COPD as compared with those without COPD was observed (Fig. 2). An improvement in QoL after 12 months was confirmed for both groups for mobility and anxiety/depression components of the EQ-5D-3L. On the contrary, an improvement in self-care was confirmed only for patients with COPD. The median VAS score at baseline [40.0 (30.0-50.0) vs. $45.0(25.0-60.0) ; p=0.83]$ and 12 months $[70.0$ (60.0-80.0) vs. $70.0(60.0-77.5) ; p=0.56]$ was comparable between groups. No difference in change of VAS from baseline to 12 months was found [25.0 (15.0-40.0) vs. 25.0 (10.0-30.0); $p=0.58]$.

\section{Discussion}

The major finding of our study is that COPD had a detrimental effect on long-term all-cause mortality in patients with severe AS scheduled for TAVI. These results were maintained after the adjustment for age and gender, while no difference in mortality between patients with and without COPD was observed after adjustment for most of the frailty indices. Furthermore, COPD was not identified as an independent predictor of long-term all-cause mortality.
Thus, effects of COPD might be partially related to coexisting comorbidities and frailty. Importantly, our findings stay in line with the previous studies reporting association between COPD and adverse outcomes after TAVI [13-15, 21, 22]. However, other studies presented contradictory results suggesting no influence of COPD on mortality after TAVI [16, 17]. For instance, in multicenter Canadian experience, the strongest predictor of late mortality was COPD followed by chronic kidney disease, chronic atrial fibrillation, and frailty [21]. A recent large metaanalysis [13] has reported negative impact of COPD on both short- and long-term all-cause mortality (30 days OR $1.43,95 \%$ CI $1.14-1.79$; > 2 years: HR $1.34,95 \%$ CI 1.12-1.61). COPD was also associated with increased short- and mid-term cardiovascular mortality (30 days OR 1.29 , 95\% CI 1.02-1.64; 1 year: HR 1.09, 95\% CI 1.02-1.17). Furthermore, patients with COPD had 2 times higher risk for AKI than patients without COPD. It has been suggested that COPD is likely to cause a reduction of renal blood flow [23], and that periprocedural impairment of heart function and the occurrence of hypoxemia and hypercapnia can lead to deterioration in renal function [13, 24]. In addition, COPD has been reported as an important predictor of stroke after TAVI [25]. In our study, only a trend towards higher risk of MI, new onset atrial fibrillation, and cerebrovascular accidents was noted in patients with COPD at 12-month follow-up. Numerically higher rates of those adverse events may suggest that those results could reach statistical significance with a large sample. Importantly, paroxysmal atrial fibrillation 
Table 3 Procedural and echocardiographic data after the procedure

\begin{tabular}{|c|c|c|c|c|}
\hline \multirow[t]{2}{*}{ Variable } & \multirow{2}{*}{$\begin{array}{l}\text { All patients } \\
(n=148)\end{array}$} & \multicolumn{2}{|c|}{ Chronic obstructive pulmonary disease } & \multirow[t]{2}{*}{$p$ value } \\
\hline & & $\begin{array}{l}\text { Absent } \\
(n=129)\end{array}$ & $\begin{array}{l}\text { Present } \\
(n=19)\end{array}$ & \\
\hline Access type & & & & 0.84 \\
\hline Transfemoral & $117(79.1 \%)$ & $102(79.1 \%)$ & $15(78.9 \%)$ & \\
\hline Transapical & $28(18.9 \%)$ & $24(18.6 \%)$ & $4(21.1 \%)$ & \\
\hline Transaortic & $2(1.4 \%)$ & $2(1.6 \%)$ & $0(0.0 \%)$ & \\
\hline Subclavian & $1(0.7 \%)$ & $1(0.8 \%)$ & $0(0.0 \%)$ & \\
\hline Device implanted & & & & 0.23 \\
\hline Corevalve / Evolut R & $29(19.6 \%)$ & $28(21.7 \%)$ & $1(5.3 \%)$ & \\
\hline Edwards Sapien & $95(64.2 \%)$ & $83(64.3 \%)$ & $12(63.2 \%)$ & \\
\hline Jena & $10(6.8 \%)$ & $7(5.4 \%)$ & $3(15.8 \%)$ & \\
\hline Lotus & $9(6.1 \%)$ & $7(5.4 \%)$ & $2(10.5 \%)$ & \\
\hline NVT & $5(3.4 \%)$ & $4(3.1 \%)$ & $1(5.3 \%)$ & \\
\hline Prosthesis size & & & & 0.35 \\
\hline $23 \mathrm{~mm}$ & $30(20.3 \%)$ & $26(20.2 \%)$ & $4(21.1 \%)$ & \\
\hline $25 \mathrm{~mm}$ & $8(5.4 \%)$ & $7(5.4 \%)$ & $1(5.3 \%)$ & \\
\hline $26 \mathrm{~mm}$ & $56(37.8 \%)$ & $52(40.3 \%)$ & $4(21.1 \%)$ & \\
\hline $27 \mathrm{~mm}$ & $8(5.4 \%)$ & $6(4.7 \%)$ & $2(10.5 \%)$ & \\
\hline $29 \mathrm{~mm}$ & $38(25.7 \%)$ & $32(24.8 \%)$ & $6(31.6 \%)$ & \\
\hline $31 \mathrm{~mm}$ & $8(5.4 \%)$ & $6(4.7 \%)$ & $2(10.5 \%)$ & \\
\hline Prosthesis size, median (IQR) [mm] & $26.0(25.0-29.0)$ & $26.0(25.0-29.0)$ & $27.0(25.5-29.0)$ & 0.33 \\
\hline Maximal transvalvular gradient, median (IQR) $[\mathrm{mmHg}]$ & $13.0(10.0-19.0)$ & $13.0(10.0-19.0)$ & $16.0(13.0-21.0)$ & 0.13 \\
\hline Median transvalvular gradient, median (IQR) $[\mathrm{mmHg}]$ & $7.4(5.1-10.0)$ & $7.0(5.0-10.0)$ & $9.0(6.0-11.0)$ & 0.12 \\
\hline Left ventricle ejection fraction, median (IQR) [\%] & $48.0(41.0-55.0)$ & $49.0(43.0-55.0)$ & $45.0(32.5-60.0)$ & 0.61 \\
\hline Aortic regurgitation & & & & 0.60 \\
\hline 0 & $84(56.8 \%)$ & $71(55.0 \%)$ & $13(68.4 \%)$ & \\
\hline 1 & $55(37.2 \%)$ & $50(38.8 \%)$ & $5(26.3 \%)$ & \\
\hline 2 & $7(4.7 \%)$ & $6(4.7 \%)$ & $1(5.3 \%)$ & \\
\hline 3 & $2(1.4 \%)$ & $2(1.6 \%)$ & $0(0.0 \%)$ & \\
\hline Radiation dose, median (IQR) [mGy] & $721.0(632.5-827.5)$ & $713.0(632.0-823.0)$ & $789.0(639.5-905.5)$ & 0.13 \\
\hline Contrast medium load, median (IQR) [ml] & $75.0(50.0-137.5)$ & $75.0(50.0-100.0)$ & $75.0(50.0-125.0)$ & 0.80 \\
\hline Fluoroscopy time, median (IQR) [min] & $13.0(12.0-15.0)$ & $13.0(12.0-15.0)$ & $13.0(12.0-14.0)$ & 0.70 \\
\hline
\end{tabular}

is quite common among patients with COPD and might be related to COPD exacerbation and treatment with theophylline [26, 27]. Also, COPD and coronary artery disease share several risk factors such as smoking habit and aging. Thus, it is expected that a considerable number of patients with COPD scheduled for TAVI also have concomitant coronary artery disease. In our study, these patients were more likely to have chronic total occlusions and incomplete revascularization of coronary arteries before TAVI. This result might be related to a higher prevalence of diabetes mellitus, previous MI and percutaneous coronary intervention in the COPD group. On the other hand, a link between COPD and systemic inflammatory response was suggested. That way COPD may hasten the progression of atherosclerotic disease as well as the destabilization of existing atherosclerotic plaques [27]. Interestingly, a systemic inflammatory response measured by elevation of C-reactive protein, interleukin-6, and interleukin- 8 levels has been shown to be associated with worse prognosis in patients undergoing TAVI [28].

Frailty and sarcopenia have been shown to be a frequent condition in elderly patients and should be considered when dealing with invasive care in the elderly [18, 29]. Importantly, both frailty and sarcopenia are common among patients with a chronic respiratory disease [30]. Also, those were shown to be associated with worse outcomes of patients with severe AS undergoing TAVI [18, 29]. In our study, the prevalence of frailty was numerically higher in patients with than without COPD and the presence of frailty was strongly associated with long-term all-cause 


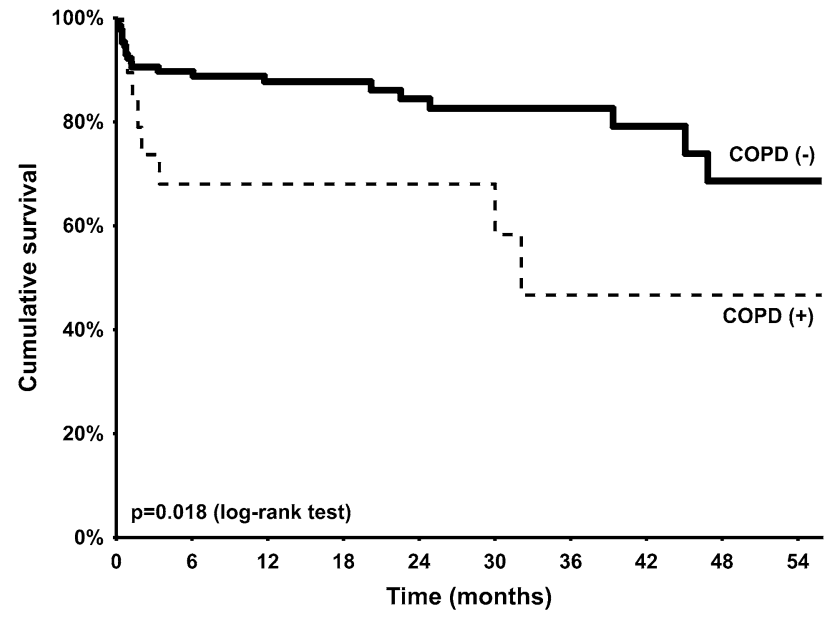

Fig. 1 Kaplan-Meier curves for survival after transcatheter valve implantation stratified by chronic obstructive pulmonary disease (COPD) status (without COPD solid line; with COPD dotted line)

mortality. On the other hand, the observed difference in mortality between patients with and without COPD was not significant after correction for frailty indices. However, in patients with COPD and severe AS, it may be difficult to distinguish the precise contribution of each pathology, when progressive dyspnea appears [31]. Also, in inoperable patients with severe AS and COPD, it seems crucial to define the border between utility and futility, when the condition is too far advanced and the risk too high even for this less invasive procedure.

Evaluation of QoL seems to be an important index as frequently not a reduction in mortality but improvement in daily life comfort is most desirable by patients themselves [9-11]. Importantly, improvement in QoL after TAVI may be higher than observed after SAVR, even with the use of minimally-invasive surgical techniques (mini-thoracotomy, mini-sternotomy) [11]. However, these benefits in terms of improvement in QoL after TAVI may be affected by the presence of comorbidities, including COPD. De Oliveira et al. [32] suggested that QoL in patients with COPD was lower than expected. Also, up to $20 \%$ of patients with COPD were on antidepressants, which had a possible influence on QoL outcomes [32]. Furthermore, increasing level of severity of COPD was associated with a significant decline in the EQ-5D VAS scores and utility scores [33]. In our study, an improvement in QoL at 12 months after TAVI was confirmed for patients both with and without COPD for mobility and anxiety/depression components of the EQ-5D-3L. On the contrary, an improvement in self-care was confirmed only for patients with COPD. No difference in VAS change during followup between groups was reported. This might suggest an equal response to TAVI in terms of QoL regardless of COPD status.

Several important limitations of the present study should be acknowledged. First, the study has all the limitations of a single-center registry. Second, patients were not stratified by the severity of COPD. It is probable that the relative impact of COPD on outcomes is dependent on disease severity. Third, symptoms control and a history of exacerbations, as well as type of treatment, were not assessed. Finally, the tools used for QoL and frailty assessment were non-disease-specific instruments. Prevalence of depression and the use of antidepressants in patients with COPD were not evaluated. However, despite several limitations, our study represents a complete analysis of consecutive patients without any exclusion criteria and with follow-up data available for all patients.
Table 4 Impact of chronic obstructive pulmonary disease and frailty indices on maximal follow-up all-cause mortality

\begin{tabular}{lcc}
\hline Variable & Age/gender-adjusted HR $(95 \%$ & $p$ value \\
& CI $)$ & $<0.001$ \\
\hline 5-meter walking test $\geq 6 \mathrm{~s}$ & $14.71(6.50-33.3)$ & 0.34 \\
Chronic obstructive pulmonary disease & $1.47(0.66-3.26)$ & $<0.001$ \\
Elderly mobility scale $<10$ points & $13.80(5.34-35.64)$ & 0.45 \\
Chronic obstructive pulmonary disease & $1.41(0.58-3.42)$ & $<0.001$ \\
Canadian Study of Health and Aging scale 5-7 points & $39.10(15.85-96.46)$ & 0.16 \\
Chronic obstructive pulmonary disease & $1.82(0.79-4.18)$ & $<0.001$ \\
Katz index $<6$ points & $13.92(6.29-30.79)$ & 0.82 \\
Chronic obstructive pulmonary disease & $1.10(0.48-2.56)$ & $<0.001$ \\
Weak grip strength & $28.84(10.54-78.87)$ & 0.006 \\
Chronic obstructive pulmonary disease & $3.09(1.37-6.95)$ & $<0.001$ \\
Identification of Seniors at Risk scale $\geq 2$ points & $5.25(2.20-12.55)$ & 0.021 \\
Chronic obstructive pulmonary disease & $2.57(1.16-5.70)$ &
\end{tabular}


Fig. 2 Proportions of patients that report either "some problems"/"extreme problems" for each category of the EQ-5D-3L at baseline and 12 months

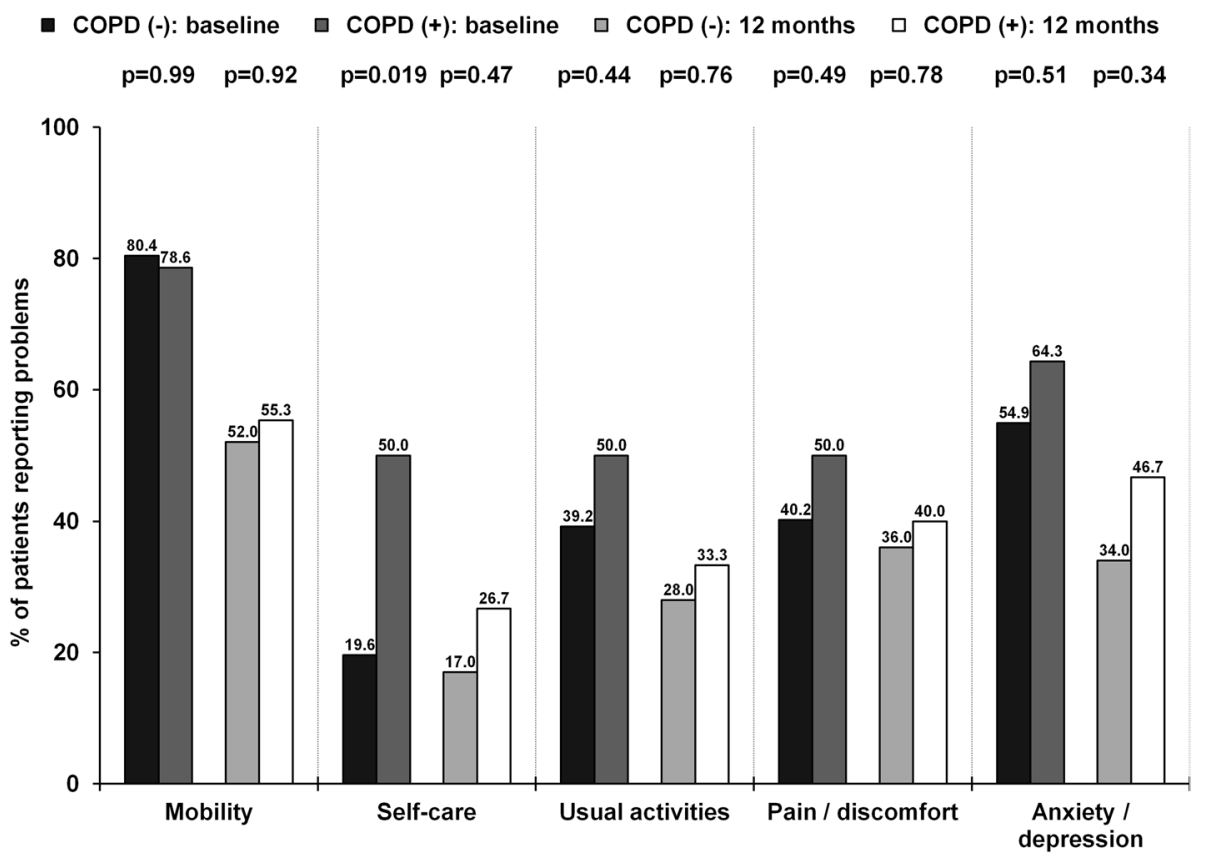

\section{Compliance with ethical standards}

Conflict of interest The authors have no conflict of interest to declare.

Ethical approval The study protocol had been approved by the ethical board of Jagiellonian University.

Statement of human and animal rights The study protocol and execution complied with the Declaration of Helsinki.

Informed consent All patients provided informed consent.

Open Access This article is distributed under the terms of the Creative Commons Attribution 4.0 International License (http://creativecommons.org/licenses/by/4.0/), which permits unrestricted use, distribution, and reproduction in any medium, provided you give appropriate credit to the original author(s) and the source, provide a link to the Creative Commons license, and indicate if changes were made.

\section{References}

1. Brown JM, O'Brien SM, Wu C et al (2009) Isolated aortic valve replacement in North America comprising 108,687 patients in 10 years: changes in risks, valve types, and outcomes in the Society of Thoracic Surgeons National Database. J Thorac Cardiovasc Surg 137:82-90. https://doi.org/10.1016/j. jtcvs.2008.08.015

2. Zlotnick DM, Ouellette ML, Malenka DJ et al (2013) Effect of preoperative pulmonary hypertension on outcomes in patients with severe aortic stenosis following surgical aortic valve replacement. Am J Cardiol 112:1635-1640. https://doi.org/10.1016/j. amjcard.2013.07.025

3. Ferguson TB Jr, Dziuban SW Jr, Edwards FH et al (2000) The STS National Database: current changes and challenges for the new millennium. Committee to Establish a National Database in
Cardiothoracic Surgery, The Society of Thoracic Surgeons. Ann Thorac Surg 69:680-691

4. Nashef SA, Roques F, Michel P et al (1999) European system for cardiac operative risk evaluation (EuroSCORE). Eur J Cardiothorac Surg 16:9-13

5. Leon MB, Smith CR, Mack M et al (2010) Transcatheter aortic-valve implantation for aortic stenosis in patients who cannot undergo surgery. N Engl J Med 363:1597-1607. https://doi. org/10.1056/NEJMoa1008232

6. Leon MB, Smith CR, Mack MJ et al (2016) Transcatheter or surgical aortic-valve replacement in intermediate-risk patients. N Engl J Med 374:1609-1620. https://doi.org/10.1056/NEJMoa1514616

7. Tokarek T, Sobczynski R, Dziewierz A et al (2015) Clinical outcomes in patients after surgical and transcatheter aortic valve replacement. Pol Arch Med Wewn 125:755-764

8. Bagienski M, Kleczynski P, Dziewierz A et al (2016) Early- and mid-term outcomes after transcatheter aortic valve implantation. Data from a single-center registry. Adv Interv Cardiol 12:122127. https://doi.org/10.5114/aic.2016.59362

9. Kleczynski P, Bagienski M, Sorysz D et al (2014) Short- and intermediate-term improvement of patient quality of life after transcatheter aortic valve implantation: a single-centre study. Kardiol Pol 72:612-616. https://doi.org/10.5603/KP.a2014.0065

10. Kleczynski P, Bagienski M, Dziewierz A et al (2016) Twelvemonth quality of life improvement and all-cause mortality in elderly patients undergoing transcatheter aortic valve replacement. Int J Artif Organs 39:444-449. https://doi.org/10.5301/ ijao. 5000521

11. Tokarek T, Siudak Z, Dziewierz A et al (2016) Assessment of quality of life in patients after surgical and transcatheter aortic valve replacement. Catheter Cardiovasc Interv 88:E80-E88. https://doi.org/10.1002/ccd.26400

12. Stoklosa P, Szymanski P, Dabrowski M et al (2015) The impact of transcatheter aortic valve implantation on left ventricular performance and wall thickness - single-centre experience. Postepy Kardiol Interwencyjnej 11:37-43. https://doi.org/10.5114/ pwki.2015.49183

13. Liao YB, He ZX, Zhao ZG et al (2016) The relationship between chronic obstructive pulmonary disease and transcatheter aortic 
valve implantation-a systematic review and meta-analysis. Catheter Cardiovasc Interv 87(Suppl 1):570-578. https://doi. org/10.1002/ccd.26443

14. Mok M, Nombela-Franco L, Dumont E et al (2013) Chronic obstructive pulmonary disease in patients undergoing transcatheter aortic valve implantation: insights on clinical outcomes, prognostic markers, and functional status changes. JACC Cardiovasc Interv 6:1072-1084. https://doi.org/10.1016/j. jcin.2013.06.008

15. Chopard R, Meneveau N, Chocron S et al (2014) Impact of chronic obstructive pulmonary disease on Valve Academic Research Consortium-defined outcomes after transcatheter aortic valve implantation (from the FRANCE 2 Registry). Am J Cardiol 113:1543-1549. https://doi.org/10.1016/j.amjcard.2014.01.432

16. Gotzmann M, Knoop H, Ewers A et al (2015) Impact of lung diseases on morbidity and mortality after transcatheter aortic valve implantation: insights from spirometry and body plethysmography. Am Heart J 170:837-842. doi: https://doi. org/10.1016/j.ahj.2015.07.011

17. Poulsen MK, Dahl JS, Kjeldsen BJ et al (2015) Impact of chronic obstructive pulmonary disease on survival and symptoms of severe aortic valve stenosis. Scand Cardiovasc J 49:4955. https://doi.org/10.3109/14017431.2015.1006671

18. Kleczynski P, Dziewierz A, Bagienski M et al (2017) Impact of frailty on mortality after transcatheter aortic valve implantation. Am Heart J 185:52-58. https://doi.org/10.1016/j.ahj.2016.12.005

19. Kappetein AP, Head SJ, Genereux P et al (2012) Updated standardized endpoint definitions for transcatheter aortic valve implantation: the Valve Academic Research Consortium-2 consensus document. EuroIntervention 8:782-795. https://doi.org/10.4244/ EIJV8I7A121

20. Vestbo J, Hurd SS, Agusti AG et al (2013) Global strategy for the diagnosis, management, and prevention of chronic obstructive pulmonary disease: GOLD executive summary. Am J Respir Crit Care Med 187:347-365. https://doi.org/10.1164/ rccm.201204-0596PP

21. Rodes-Cabau J, Webb JG, Cheung A et al (2012) Long-term outcomes after transcatheter aortic valve implantation: insights on prognostic factors and valve durability from the Canadian multicenter experience. J Am Coll Cardiol 60:1864-1875. https://doi. org/10.1016/j.jacc.2012.08.960

22. Moat NE, Ludman P, de Belder MA et al (2011) Long-term outcomes after transcatheter aortic valve implantation in high-risk patients with severe aortic stenosis: the U.K. TAVI (United Kingdom Transcatheter Aortic Valve Implantation) Registry. J Am Coll Cardiol 58:2130-2138. https://doi.org/10.1016/j.jacc.2011.08.050
23. Gluhovschi G, Velciov S, Petrica L, Gluhovschi C (2014) Aspects of renal-pulmonary pathogenic replationships in chronic kidney disease and chronic pulmonary diseases - a less-known connection. Rom J Intern Med 52:68-77

24. Anand IS, Chandrashekhar Y, Ferrari R et al (1992) Pathogenesis of congestive state in chronic obstructive pulmonary disease. Studies of body water and sodium, renal function, hemodynamics, and plasma hormones during edema and after recovery. Circulation 86:12-21

25. Stortecky S, Windecker S, Pilgrim T et al (2012) Cerebrovascular accidents complicating transcatheter aortic valve implantation: frequency, timing and impact on outcomes. EuroIntervention 8:62-70. https://doi.org/10.4244/EIJV8I1A11

26. Rusinowicz T, Zielonka TM, Zycinska K (2017) Cardiac arrhythmias in patients with exacerbation of COPD. Adv Exp Med Biol. https://doi.org/10.1007/5584_2017_41

27. Dziewierz A, Siudak Z, Rakowski T et al (2010) Relationship between chronic obstructive pulmonary disease and in-hospital management and outcomes in patients with acute myocardial infarction. Kardiol Pol 68:294-301

28. Sinning JM, Scheer AC, Adenauer V et al (2012) Systemic inflammatory response syndrome predicts increased mortality in patients after transcatheter aortic valve implantation. Eur Heart J 33:1459_ 1468. https://doi.org/10.1093/eurheartj/ehs002

29. Saji M, Lim DS, Ragosta M et al (2016) Usefulness of psoas muscle area to predict mortality in patients undergoing transcatheter aortic valve replacement. Am J Cardiol 118:251-257. https://doi. org/10.1016/j.amjcard.2016.04.043

30. Bone AE, Hepgul N, Kon S et al (2017) Sarcopenia and frailty in chronic respiratory disease. Chron Respir Dis 14:85-99. https:// doi.org/10.1177/1479972316679664

31. Hawkins NM, Petrie MC, Jhund PS et al (2009) Heart failure and chronic obstructive pulmonary disease: diagnostic pitfalls and epidemiology. Eur J Heart Fail 11:130-139. https://doi.org/10.1093/ eurjhf/hfn013

32. de Oliveira JC, de Carvalho Aguiar I, de Oliveira Beloto AC et al (2013) Clinical significance in COPD patients followed in a real practice. Multidiscip Respir Med 8:43. https://doi. org/10.1186/2049-6958-8-43

33. Rutten-van Molken MP, Oostenbrink JB, Tashkin DP et al (2006) Does quality of life of COPD patients as measured by the generic EuroQol five-dimension questionnaire differentiate between COPD severity stages? Chest 130:1117-1128. https:// doi.org/10.1378/chest.130.4.1117 